\title{
Rare Case of Sigmoid-Perianal Fistula due to Sigmoid Diverticular Disease: Report of a Case and Review of Literature
}

\author{
Kaushik Kumara, b, Polbert Diaz $^{\mathrm{a}}$
}

\begin{abstract}
Diverticular disease of colon is a common problem of western countries and its incidence usually increases with age. The natural history of diverticular disease is usually benign with only few patients developing episode of acute diverticulitis. Some patients may develop more complicated courses like hemorrhage, obstruction, stricture or fistulas. While colovesical and colovaginal fistulas are the common types of fistula, perianal fistula due to diverticular disease is an extremely rare complication of diverticular disease. We report a case of sigmoid-perianal fistula due to diverticular disease in a 53-year-old female patient without any signs or symptoms of sigmoid diverticulitis. Diagnosis was established in early course of disease by computed tomogram (CT) scan and a one-stage anterior resection was performed without any complication. We also performed literature review for similar cases and how they were managed.
\end{abstract}

Keywords: Diverticular disease; Diverticulitis; Fistula; Perianal fistula

\section{Introduction}

Diverticular disease of colon is common problem of western countries with prevalence of $20-60 \%$ and the incidence usually increases with age. The natural history of diverticular disease is usually benign with only $15-20 \%$ patients developing episodes of acute diverticulitis. Some patients may present with other complications like hemorrhage, obstruction, stricture or fistulas. Colovesical and colovaginal fistulas are the common types of fistula due to diverticular disease, while perianal fistulas due to diverticular disease are an extremely rare complication of diverticular disease. We report a case of sigmoid-perianal fistula due to diverticular disease in a 53-year-old female

Manuscript accepted for publication January 29, 2015

${ }^{a}$ General Surgery Department, St Stephen's Hospital, 182-198, Nissen Street, Urraween, Qld 4655, Australia

${ }^{\text {b} C o r r e s p o n d i n g ~ A u t h o r: ~ K a u s h i k ~ K u m a r, ~ 3, ~ K e n n e d y ~ C o u r t, ~ U r r a w e e n, ~ Q l d ~}$ 4655, Australia. Email: drkaushik1977@gmail.com

doi: http://dx.doi.org/10.14740/jmc2065w patient without any obvious signs or symptoms of sigmoid diverticulitis. Diagnosis was established with imaging study in early course of disease due to non-healing of wound. A onestage anterior resection was performed without any complication. We also review literatures to find similar reported cases and how they were managed.

\section{Case Report}

A 53-year-old female patient was referred by general practitioner (GP) to our clinic for management of a non-healing leftsided perianal wound. She had a small perianal lump with some redness for few weeks that burst open by itself and drained purulent fluid. Her GP did a minor debridement of the wound and started her on antibiotics and daily dressings. The wound did not heal completely after 2 weeks of dressing and antibiotics, so her GP organized a computed tomogram (CT) scan of abdomen and pelvis that revealed diverticulosis of sigmoid colon with a long fistulous tract going towards the perianal wound. On examination, her vitals and abdominal examinations were unremarkable. Rectal examination did not reveal any obvious abnormality apart from a small non-healing wound on left side of the anal opening about $2-3 \mathrm{~cm}$ from the anal verge without any acute cellulitis or new abscess. All her blood reports including WBC, CRP and tumor markers were also normal.

The patient had occasional left lower quadrant abdominal pain over the past couple of months but no distinct episode of diverticulitis which required antibiotics or hospitalization. She denied any history of trauma, per rectal bleeding, altered bowel habits, weight loss, anorexia, fever, vomiting or pneumaturia. There was no family history of inflammatory bowel disease (IBD). She had a colonoscopy 14 years prior due to a family history of bowel cancer that revealed diverticulosis of the sigmoid colon.

A CT fistulogram was organized which showed a long fistulous tract extending from the left perianal region to a diverticulum above the recto-sigmoid junction; contrast was seen entering the sigmoid loop (Fig. 1). A colonoscopy was attempted but due to an acute angle at the recto-sigmoid junction, it was not possible to pass the scope beyond that point. She was put on oral antibiotics and daily packing of the wound to keep the fistula open until a bowel resection could be done. She underwent elective laparoscopic-assisted sigmoid resec- 


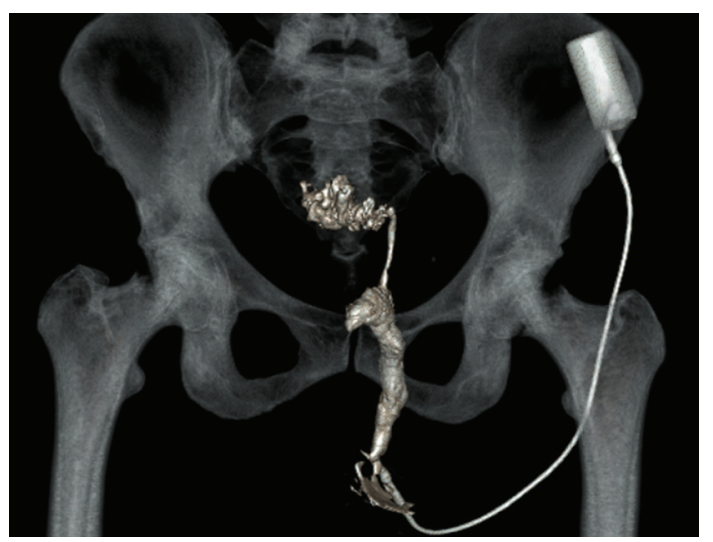

Figure 1. Reconstructed CT fistulogram image of sigmoid-perianal fistula.

tion. The abdominal cavity was "clean" with no infectious process visible. The distal sigmoid and rectosigmoid junction were found densely adherent to the left lateral pelvic wall (Fig. 2 ). The proximal sigmoid and descending colon were mobilized laparoscopically, including division of the vascular pedicle, but due to technical difficulty of dissection owing to the dense adhesions, we converted to a lower midline laparotomy which allowed a safe mobilization of the recto-sigmoid. The sigmoid loop was densely stuck in the pelvis; the left ovary was adhered to it as well. No abscess cavity was encountered in the operation, suggesting that the diverticulum had sealed off. The rectum was noted to be normal. High anterior resection was performed; the upper rectum was transacted using the echelon stapler (Ethicon). Primary colo-rectal anastomosis was achieved using a $29 \mathrm{~mm}$ circular stapler (Ethicon). The post-operative course was uneventful and the patient recovered quite rapidly, bowel function returning on the evening of day one. She was discharged home with full bowel function on the third post-operative day. The perianal wound, which was simply curetted and packed with Aquacel rope, healed completely within 2 weeks. Histology confirmed diverticular disease with inflammation of the colon. The patient is booked for completion colonoscopy in 6 months' time.

\section{Discussion}

Diverticular disease is a common problem in western countries. Most of the patients with diverticular disease remain asymptomatic; others develop a variable course. The most common complication is diverticulitis, affecting about 15$20 \%$ of patients with diverticulosis and it is due to microperforation of a diverticulum. Others may develop complications like stricture, obstruction, hemorrhage and fistulas. Study by Vasilevsky et al suggested that colovesical fistula (48\%) and colovaginal $(44 \%)$ fistulas are the common types of fistulas [1], while, coloenteric, colocutaneous, coloovarian, colouterine, coloseminal, ureterocolic and coloumbilical fistulas are relatively rare. The colocutaneous fistula usually occurs after previous resection of colon for diverticular disease or percutaneous drainage of diverticular abscess [2]. A perianal fistula due to diverticular disease is an extremely rare complication

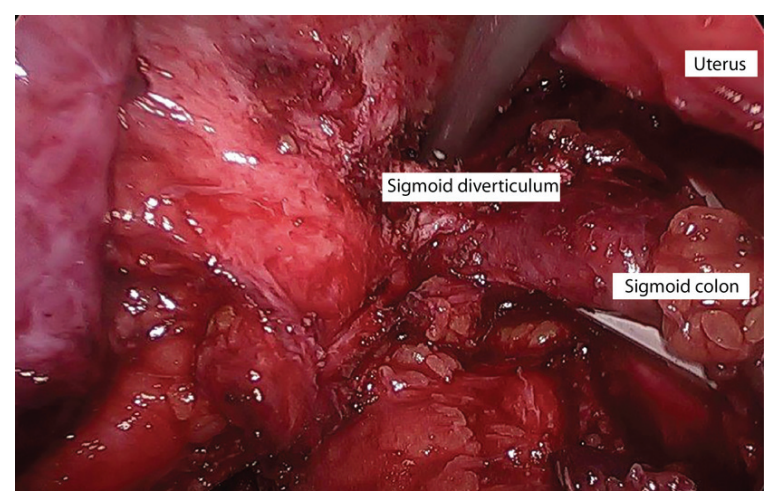

Figure 2. Laparoscopic image of sigmoid diverticulum densely adherent to left pelvic side wall.

and our review of literature found only six such reported cases [2-7].

A fistula usually results from a colon perforation and paracolic abscess formation with extension into adjacent structures. The infection from sigmoid diverticultis can spread along the rectum, penetrating the levator ani muscle into the perineum and perianal region, as suggested by Stahlgren and Thabit as one of the seven modes of spread paracolic abscess [8].

Most patients with complicated diverticular disease have some sort of symptoms such as abdominal pain, vomiting, nauseas, diarrhoea, constipation, fever, weight loss and rectal bleeding. Patients with colovesical fistula may present with pneumaturia. However, the patient can be completely asymptomatic or may have mild symptoms that may be attributed to other diseases, like irritable bowel syndrome as in our case. Very elderly patients and immunocompromised patients may not show any obvious signs even in complicated disease.

Studies have reported that the first episode of diverticulitis is usually the worst episode and with subsequent episodes the risk of septic peritonitis decreases. In a study by BroderickVilla et al, the risk of first recurrence was $13.3 \%$ and second recurrence was 3.9\% after the first episode of diverticulitis [9].

The real problem is differentiation of diverticulitis from IBD when presenting features are confusing. Also possible underlying malignancy would also need to be ruled out. The common practice is to perform colonoscopy 6 - 8 weeks after an acute episode of diverticulitis.

CT scan is a very sensitive test for the diagnosis of diverticular disease. It is non-invasive and readily available. It helps to define the extent and detects complications of diverticular disease. CT grading of diverticular disease such as suggested by Ambrosetti et al [10] serves as useful guide for planning management. CT or ultrasound-guided aspiration of diverticular abscess has been treatment of choice when patient is hemodynamically stable and the position and size of the abscess is suitable for the procedure. In our case, the CT scan was the first test to indicate the sigmoid-perianal fistula, which was subsequently confirmed with a CT fistulogram. MRI is gaining its popularity, at least in complicated disease, due to lack of ionizing radiation and has matching sensitivity and specificity of a CT scan. It gives better information in cases of fistula. However, its usefulness is limited due to lack of availability in 
smaller centers, cost factor, and the need of experienced radiologists [11].

Simple diverticulitis and also some complicated diverticulitis cases are often managed conservatively with intravenous fluids, analgesia, antibiotics and aspiration of the abscess, if required. The role of surgery in the emergency setting is limited to the hemodynamically unstable patient with purulent or feculent peritonitis or closed loop large bowel obstruction. While the role of elective surgery in diverticular disease has changed over time, only a few patients with recurrent acute episodes or complicated cases require surgery. Laparoscopic surgery has gained popularity and is safely done in the elective setting with minimal morbidity and mortality. One stage resection and anastomosis is the standard of treatment in the elective setting; two-stage surgery is required in some complicated cases, especially in the emergency setting. A randomized controlled trial in 2009 confirmed the superiority of laparoscopic surgery for diverticular diseases with $15.4 \%$ reduction in major morbidity, less pain, shorter hospitalization, and improved quality of life [12].

Patients with fistulas usually require surgery [13]. Complicated fistula due to diverticular disease may sometimes require a staged procedure with an initial diverting colostomy with resection anastomosis in subsequent surgery, thus allowing the fistula to heal and control infection, as in the case reported by Harris and Marshak [3]. In our case, one-stage surgery was done without any operative or post-operative complication. Most of the colonic mobilization was achieved laparoscopically; however, due to dense adhesions of the distal sigmoid to the pelvic side wall, the procedure was converted to an open laparotomy. In previously reported similar cases, three-stage surgeries were required in four cases [2-4, 6] and one-stage surgery was performed in two cases $[5,7]$.

Our case is unique in that the patient never had any episode of diverticulitis and the first presentation was with a perianal abscess. The patient was not immunocompromised and had no previous abdominal or pelvic surgery. A one-stage operation was performed without any complications. In previously reported similar cases, three-stage surgeries were required in four cases $[2-4,6]$ and one-stage surgery was performed in two cases $[5,7]$. The diagnoses of sigmoid-perianal fistula was made relatively early in course of disease with CT scan and we emphasize importance of early imaging study particularly in elderly patients with non-healing fistulas to rule out associated problems like colonic diverticular problem or malignancy, as delay in diagnosis may result in delay in proper surgical treatment and increases morbidity.

\section{Conclusion}

Diverticulitis can present in various ways, from episodes of self-limiting, uncomplicated diverticulitis, to a very complicated case such as sigmoid-perianal fistula. In the case of a non-healing perianal fistula, a long fistulous tract communicating with the sigmoid due to diverticular disease should be kept in mind and needs to be excluded by imaging early in course of disease. A one-stage operation for such a fistulous tract can be performed successfully, provided there is no active abscess around the sigmoid colon. This can allow for healing of the fistula without additional need for surgery for the fistula track itself.

\section{Competing Interest}

The authors declare that they have no competing interests.

\section{References}

1. Vasilevsky CA, Belliveau P, Trudel JL, Stein BL, Gordon $\mathrm{PH}$. Fistulas complicating diverticulitis. Int J Colorectal Dis. 1998;13(2):57-60.

2. Colcock BP, Stahmann FD. Fistulas complicating diverticular disease of the sigmoid colon. Ann Surg. 1972;175(6):838-846.

3. Harris AI, Marshak RH. The radiology corner: Longitudinal fistulous tract of the colon and a perianal fistula in diverticulitis. Am J Gastroenterol. 1976;66(5):487-491.

4. Loeb PM, Berk RN, Saltzstein SL. Longitudinal fistula of the colon in diverticulitis. Gastroenterology. 1974;67(4):720-724.

5. Weyand G, Rinast E, Englert A, Houf M. [Sigmoidoperianal fistula associated with diverticulitis and $\mathrm{Cul}$ de Sac situation--diagnostics and therapy]. Zentralbl Chir. 2002;127(7):622-625.

6. Kratzer GL, Hernandez J. Diverticulitis of the colon in a community hospital. Dis Colon Rectum. 1970;13(3):231234.

7. Fujii T, Sutoh T, Morita H, Katoh T, Yajima R, Yamaguchi $\mathrm{S}$, Tsutsumi S, et al. Perianal abscess and sigmoidocutaneous fistula due to diverticulitis. Am Surg. 2012;78(2):6263.

8. Stahlgren LH, Thabit G. Subcutaneous Emphysema: An Important Sign of Intra-abdominal Abscess. Ann Surg. 1961;153(1):126-133.

9. Broderick-Villa G, Burchette RJ, Collins JC, Abbas MA, Haigh PI. Hospitalization for acute diverticulitis does not mandate routine elective colectomy. Arch Surg. 2005;140(6):576-581; discussion 581-573.

10. Ambrosetti P, Chautems R, Soravia C, Peiris-Waser N, Terrier F. Long-term outcome of mesocolic and pelvic diverticular abscesses of the left colon: a prospective study of 73 cases. Dis Colon Rectum. 2005;48(4):787-791.

11. Heverhagen JT, Sitter H, Zielke A, Klose KJ. Prospective evaluation of the value of magnetic resonance imaging in suspected acute sigmoid diverticulitis. Dis Colon Rectum. 2008;51(12):1810-1815.

12. Klarenbeek BR, Veenhof AA, Bergamaschi R, van der Peet DL, van den Broek WT, de Lange ES, Bemelman WA, et al. Laparoscopic sigmoid resection for diverticulitis decreases major morbidity rates: a randomized control trial: short-term results of the Sigma Trial. Ann Surg. 2009;249(1):39-44.

13. Frattini J, Longo WE. Diagnosis and treatment of chronic and recurrent diverticulitis. J Clin Gastroenterol. 2006;40(Suppl 3):S145-149. 